\title{
Vertical Nanowire Array-Based Light Emitting Diodes
}

\author{
Elaine Lai, Woong Kim, and Peidong Yang $(\bowtie)$ \\ Department of Chemistry, University of California, Berkeley, CA 94720, USA \\ Molecular Foundry, Material Sciences Division, Lawrence Berkeley National Laboratory, Berkeley, CA 94720, USA \\ Received: 5 May 2008/ Revised: 22 June 2008/Accepted: 22 June 2008 \\ CTsinghua Press and Springer-Verlag 2008
}

\begin{abstract}
Electroluminescence from a nanowire array-based light emitting diode is reported. The junction consists of a p-type GaN thin film grown by metal-organic chemical vapor deposition (MOCVD) and a vertical n-type $\mathrm{ZnO}$ nanowire array grown epitaxially from the thin film through a simple low temperature solution method. The fabricated devices exhibit diode like current-voltage behavior. Electroluminescence is visible to the human eye at a forward bias of $10 \mathrm{~V}$ and spectroscopy reveals that emission is dominated by acceptor to band transitions in the p-GaN thin film. It is suggested that the vertical nanowire architecture of the device leads to waveguided emission from the thin film through the nanowire array.
\end{abstract}

\section{KEYWORDS}

$\mathrm{ZnO}$ nanowire, electroluminescence, LED, waveguiding

\section{Introduction}

GaN and $\mathrm{ZnO}$ are promising materials in the field of short wavelength optoelectronics due to their inherently wide and direct bandgaps, $3.39 \mathrm{eV}$ and $3.37 \mathrm{eV}$, respectively [1,2]. Nanostructures based on these semiconductors offer the added benefit of material quality leading to improved device efficiency. The benefits of nanowire-based photonics have been assessed [3] and optoelectronic devices were realized early in the field [4]; however, research in the area of wide bandgap materials is less mature due to limitations in material growth quality as well as difficulties in growing p-type $\mathrm{ZnO}$ due to its intrinsically n-type nature $[2,5]$. Recent achievements in p-type GaN thin film growth [6] and reliable growth of aligned, high quality $\mathrm{ZnO}$ nanostructures [7] have opened the arena to novel wide-bandgap optoelectronic devices. In addition, $\mathrm{ZnO}$ and $\mathrm{GaN}$ are well suited materials because of the low lattice mismatch of about $1.9 \%$ in their wurtzite crystal structures [8].

In this paper, we present a nanowire-based light emitting diode (LED) consisting of a p-type GaN thin film and an n-type $\mathrm{ZnO}$ vertical nanowire array. The vertical nanowire array offers a number of potential advantages over the conventional thin film architecture. Firstly, the $\mathrm{ZnO}$ nanowire array is grown directly on the $\mathrm{p}-\mathrm{GaN}$ thin film using a low temperature solution procedure. This is a simple and low-cost process towards making functional $\mathrm{p}^{-}$ $n$ junctions. Secondly, the vertical nanowire array creates natural waveguiding cavities where part or all of the emission can be manipulated to travel to the top of the device, an improvement towards better extraction efficiency. Waveguiding has already

Address correspondence to p_yang@berkeley.edu 
been demonstrated in $\mathrm{ZnO}$ nanowires under photo excitation $[9,10]$.

Hybrid nanowire/thin film based LEDs have been reported previously. $\mathrm{ZnO}$ nanowire arrays have been fabricated on p-type GaN thin films [11-13] and p-type Si substrates [14]. Hybrid inorganic/organic LEDs have also been studied, employing $\mathrm{ZnO}$ nanowire arrays and poly(3,4-ethylene-dioxythiophene): poly(styrene-sulfonate) (PEDOT:PSS) thin films [15, 16] and further work has succeeded in making the devices flexible [17]. There have also been reports of electroluminescence from $\mathrm{n}-\mathrm{ZnO}$ single nanowirebased diodes on p-Si substrates [18] as well as n-GaN single nanowire-based diodes on p-GaN thin films [19, 20] and p-Si substrates [21].

This report demonstrates a solution-based growth of a $\mathrm{ZnO}$ nanowire array on a p-type $\mathrm{GaN}$ thin film. The results also suggest possible waveguiding behavior of the electroluminescence by the vertical nanowire array device architecture.

\section{Experimental}

A p-GaN thin film was grown on a c-plane sapphire substrate $\left(\mathrm{Al}_{2} \mathrm{O}_{3}\right)$ through metal-organic chemical vapor deposition (MOCVD). The dopant source was magnesium and following deposition, a post-anneal of $20 \mathrm{~min}$ at $800{ }^{\circ} \mathrm{C}$ was utilized to ionize the dopants. The resulting $1 \mu \mathrm{m}$ thin film had a hole concentration of $4.5 \times 10^{17} / \mathrm{cm}^{3}$ and hole mobility of $12 \mathrm{~cm}^{2} / \mathrm{Vs}$ as determined by a four-point Hall measurement. A low temperature solution growth method was then used to form a vertical $\mathrm{ZnO}$ nanowire array on top of the thin film. The growth method was the hydrolysis of a zinc salt in water. The substrate was suspended in solution for 2 hours at $95^{\circ} \mathrm{C}$ affording $2 \mu \mathrm{m}$ tall $\mathrm{ZnO}$ nanowires. The procedure was repeated two more times resulting in nanowire lengths of around 5-6 $\mu \mathrm{m}$. Details of this growth method are described elsewhere [7]. nanowire array
The resulting nanowire array is entirely vertical with nominal diameters of 100-600 nm (Fig. 1). The structure is wurtzite hexagonal as evident by the hexagonal cross sections shown in Fig. 1(a). The nanowires are believed to be epitaxially grown from the $\mathrm{p}-\mathrm{GaN}$ thin film because there is a good lattice match between the two materials. After nanowire growth, the substrate was then spin coated with poly(methyl methacrylate) (PMMA) to protect the nanowires and to form a buffer layer between the $\mathrm{p}-\mathrm{GaN}$ thin film and eventual nanowire metal contacts (Fig. 2). An oxygen plasma etch was used to remove the PMMA from the tips of the nanowires in order to allow them to form effective ohmic contacts. $\mathrm{Ni} / \mathrm{Au}$ contacts were thermally evaporated onto the p-GaN thin film and Ti/ Au contacts onto the exposed $\mathrm{ZnO}$ nanowire tips.

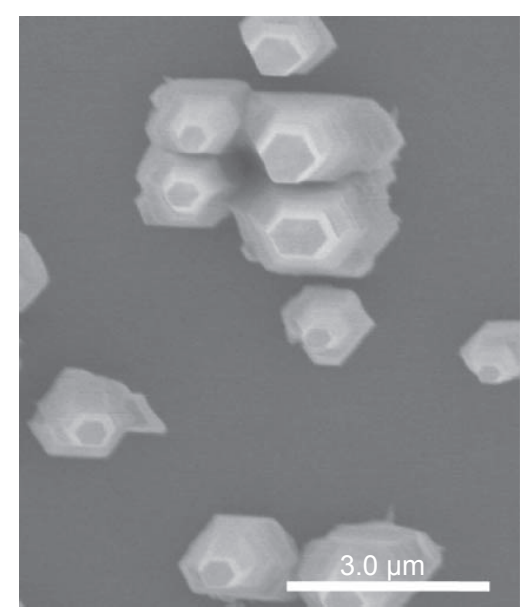

(a)

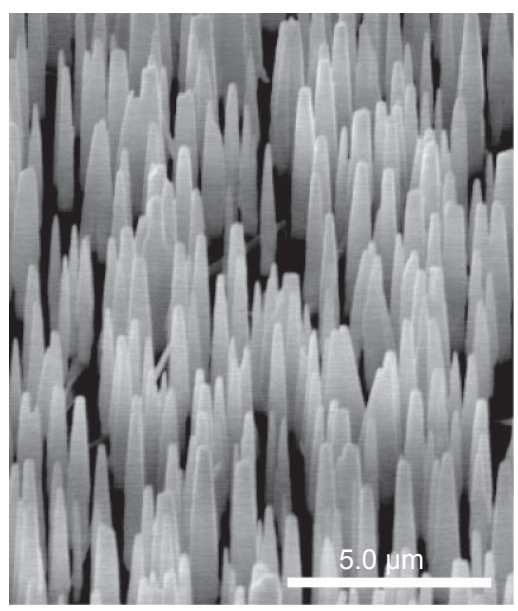

(c)

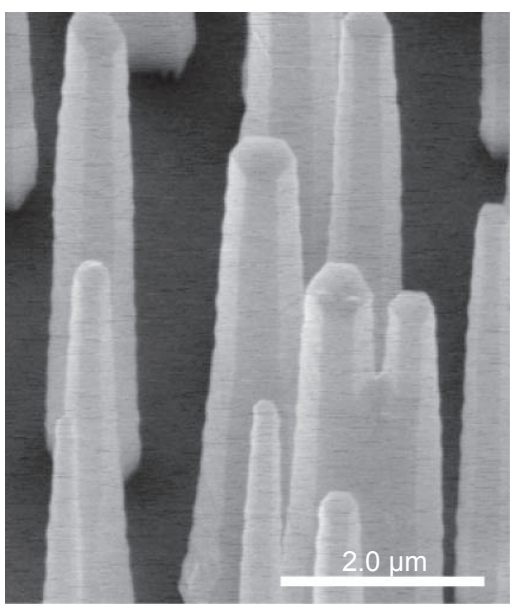

(b)

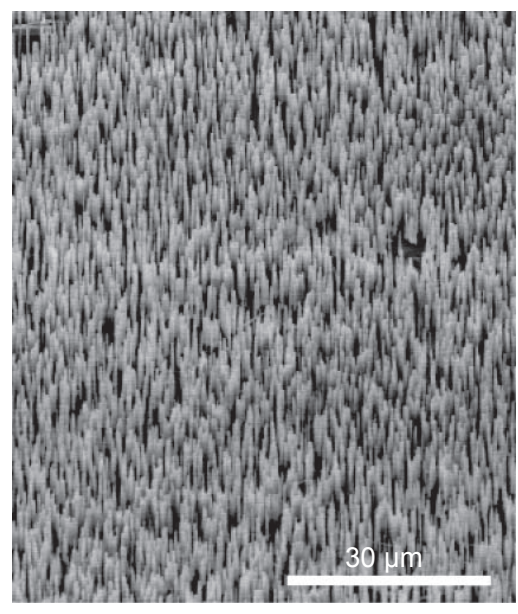

(d)

Figure 1 (a) Top down and (b)-(d) $45^{\circ}$ tilted SEM images of solution-grown ZnO 


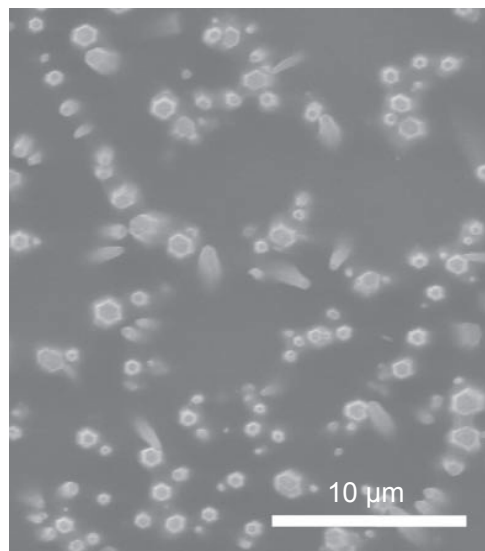

(a)

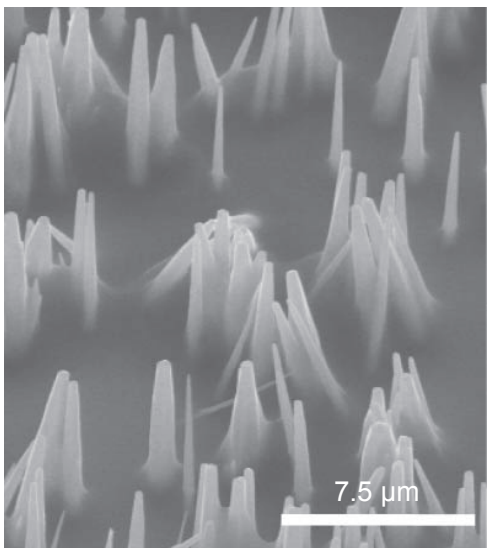

(b)

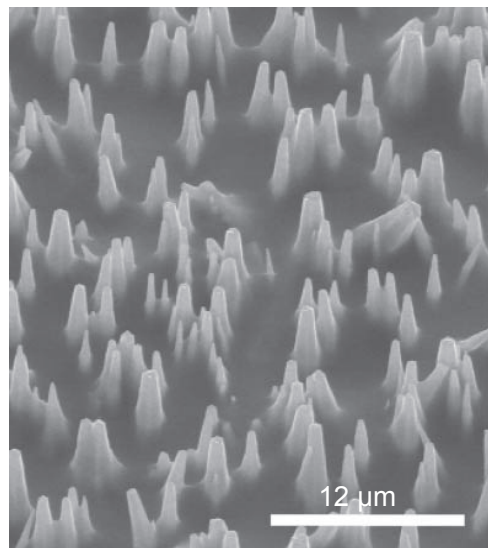

(c)

Figure 2 (a) Top down and (b), (c) $45^{\circ}$ tilted SEM images of solution-grown ZnO nanowires after spin coating of a PMMA insulating layer

\section{Results and discussion}

The resulting devices displayed electroluminescence visible to the human eye when subjected to a forward bias over $10 \mathrm{~V}$. The emitted light appeared to originate completely or perhaps predominantly from the p-type GaN thin film at the junction between the p-type thin film and n-type nanowire array (Fig. 3(a)). The localization of the electroluminescence at the $p-n$ junction gives evidence that the phenomenon is truly injection electroluminescence in which luminescence occurs from the recombination of minority carriers that were injected across the junction.

Almost all the devices showed diode like currentvoltage behavior which demonstrates proper $\mathrm{p}-\mathrm{n}$ junction formation with minimal contribution from contact resistances. The rectifying behavior is shown in Fig. 3(b). As further verification, linear current-voltage behavior was observed when electrodes were probed simply across the p-GaN thin film layer (the two lefthand metal contacts in Fig. 3(a)). Furthermore, backto-back reverse rectifying current-voltage behavior was indeed observed when electrodes were probed across the nanowire array (the two right-hand metal contacts in Fig. 3(a)).

Room temperature photoluminescence (PL) spectra of the device were collected by means of excitation with a 325-nm HeCd continuous-wave laser. Emission was collected by a Nikon microscope coupled by multimode fiber to a spectrometer with a liquid nitrogen cooled silicon charge coupled device (CCD). As the spot size of the beam at the sample was around
$100 \mu \mathrm{m}$ in diameter, it was possible to localize the excitation area. PL was observed from the bare $\mathrm{p}-\mathrm{GaN}$ thin film region of the device as well as from the $\mathrm{n}-\mathrm{ZnO}$ nanowire array region of the device (Fig. 3(c)). The emission from the $\mathrm{p}-\mathrm{GaN}$ thin film is red-shifted from the bandgap to around $430 \mathrm{~nm}$. This shift is well known and is commonly observed in Mg-doped GaN due to dominant band to acceptor transitions [22].

PL spectra collected from the nanowire array region revealed three peaks. The first peak at around $380 \mathrm{~nm}$ corresponds to interband transitions in the $\mathrm{ZnO}$ material. The second peak at around $430 \mathrm{~nm}$ results from the $\mathrm{p}-\mathrm{GaN}$ thin film directly below the nanowire array. The third broad peak centered around $600 \mathrm{~nm}$ is a "yellow peak" commonly seen in $\mathrm{ZnO}$ materials and is attributed to defects, perhaps due to interstitial oxygen ions [7].

Room temperature electroluminescence (EL) was collected by electrically stimulating the device using a Keithley voltage source and emission was detected in the same fashion as the PL measurements. Figure 3(d) shows spectra generated at $5 \mathrm{~V}$ increments between 0 and $25 \mathrm{~V}$. Electroluminescence is detectable by the naked eye at $10 \mathrm{~V}$. The emission generated is believed to be a result of a combination of the various transitions observed in the PL spectra. The dominant peak is centered at $\sim 410 \mathrm{~nm}$ and thus the electroluminescence is believed to be dominated by acceptor to band transitions in the p-GaN thin film and is blue shifted by contributions of luminescence from the interband transitions in the nanowires and perhaps interband transitions in the thin film as well. 


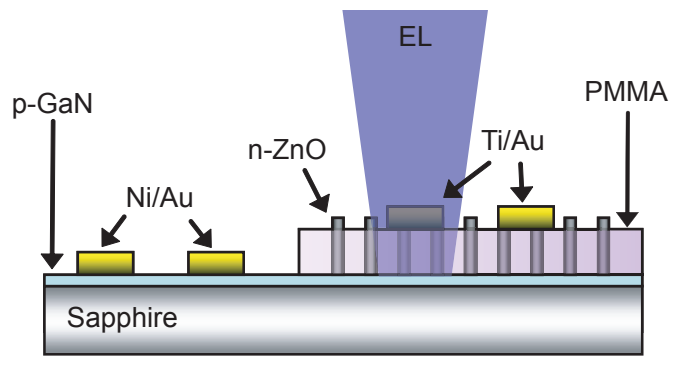

(a)

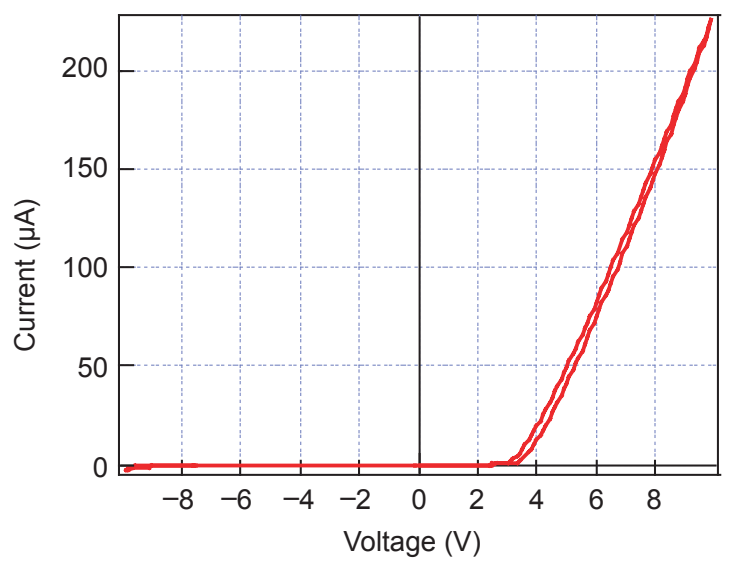

(b)

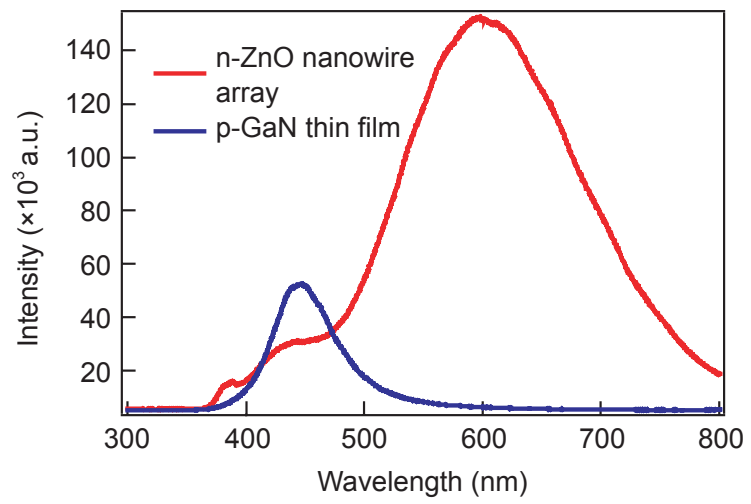

(c)

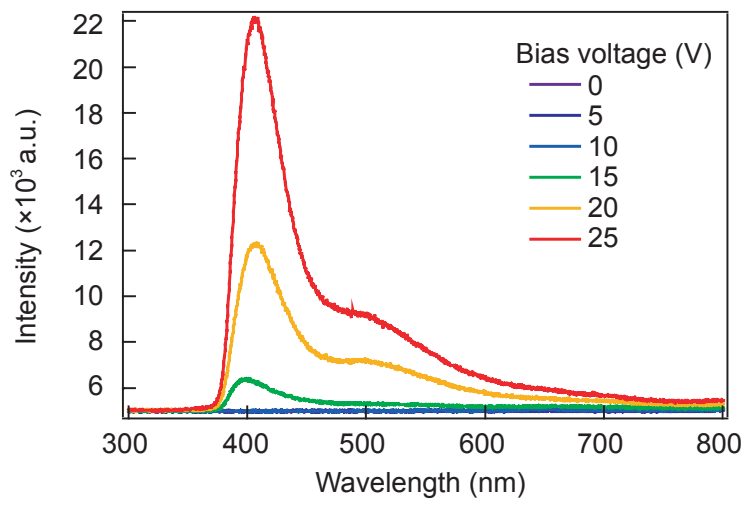

(d)

Figure 3 Solution-grown-ZnO nanowire LED device: (a) schematic view of device and location of electroluminescence; (b) I-V curves; (c) photoluminescence spectra; (d) electroluminescence spectra
The relatively low contribution from the defect peak suggests that the deep level traps may be saturating, allowing interband transitions to dominate.

An additional observation is that the change in EL intensity per unit voltage step increases as the voltage increases. This behavior is consistent with the band bending model of a $\mathrm{p}-\mathrm{n}$ junction. As voltage increases, the band bending between the $p$ and $n$ materials is reduced; therefore, the number of carriers able to traverse the junction increases. In contrast, the increase in the defect peak per voltage step is not nearly as substantial. As applied voltage increases therefore, band-to-band transitions increasingly dominate over defect-related transitions.

One of the advantages of using a vertical nanowire array over a thin film is the potential for waveguided emission, and therefore the potential for improved extraction efficiency. The natural unseeded epitaxial growth of the $\mathrm{ZnO}$ nanowires from the $\mathrm{p}-\mathrm{GaN}$ thin film yielded fairly large diameters around 100-600 nm. An ideal single mode waveguide cavity would be around $200 \mathrm{~nm}$ for a $\mathrm{ZnO}$ material surrounded by a medium of PMMA in this wavelength regime [23]. For the waveguiding experimental setup, a modified EL setup was used. The collecting optical fiber was placed directly above the luminescence site at angles ranging from $0^{\circ}$ to $90^{\circ}$ from the normal (Fig. 4(a)). At every measurement, the radial position of the end of the optical fiber was exactly $1 \mathrm{~cm}$ from the surface of the device. Figure 4(c) shows electroluminescence spectra at various angles from the normal and Fig. 4(d) shows the correlation between normalized integrated EL intensity for the various positions.

The strongest emission intensity was observed at $0^{\circ}$ from normal (directly on top of the device), and the intensity rapidly decays as detection angle increases. This observation suggests that part of the junction emission might be directly waveguided to the tip of the nanowires. A similar setup has been investigated previously, employing a vertical $\mathrm{ZnO}$ nanorod array as a waveguiding mechanism for emission from a traditional GaN-based multiple quantum well (MQW) thin film LED [24, 25]. Increases of 50\% and $100 \%$ in light output at applied currents of 20 and $50 \mathrm{~mA}$, respectively, were reported by An et al. [24] whilst Chiu et al. reported a $38.4 \%$ light output increase for 


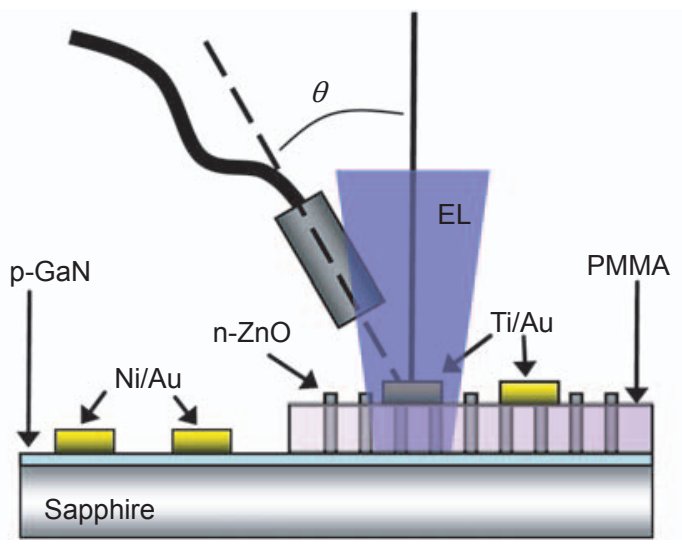

(a)

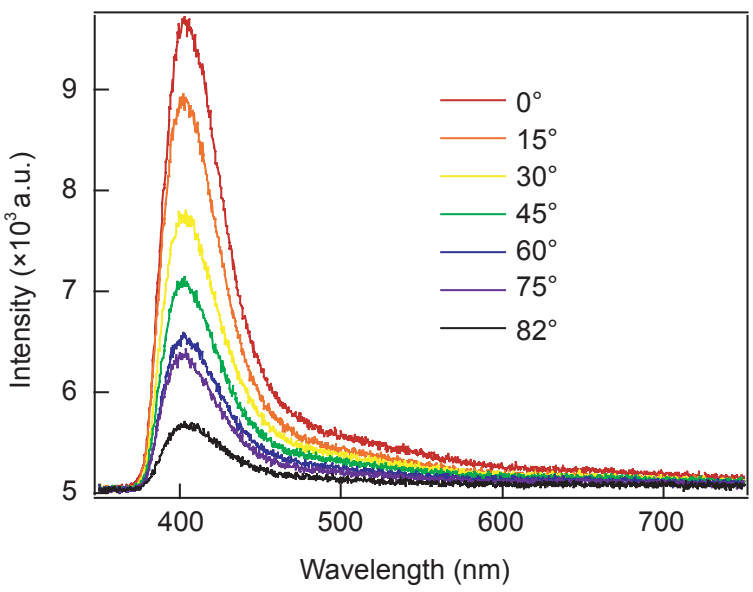

(c)

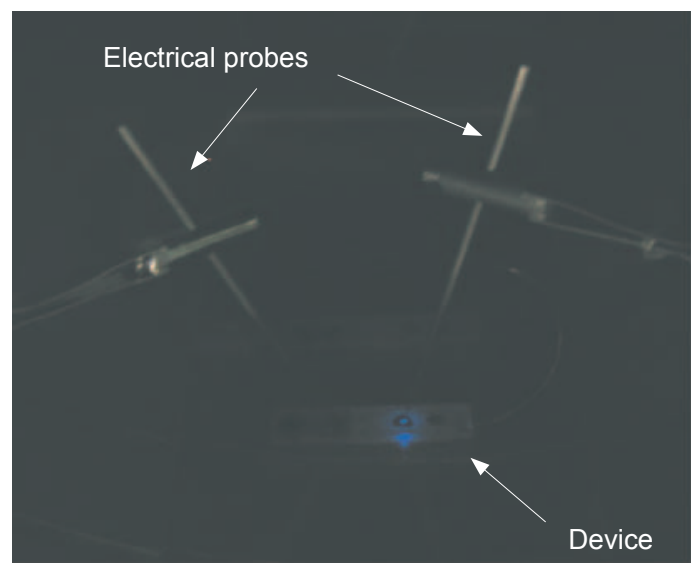

(b)

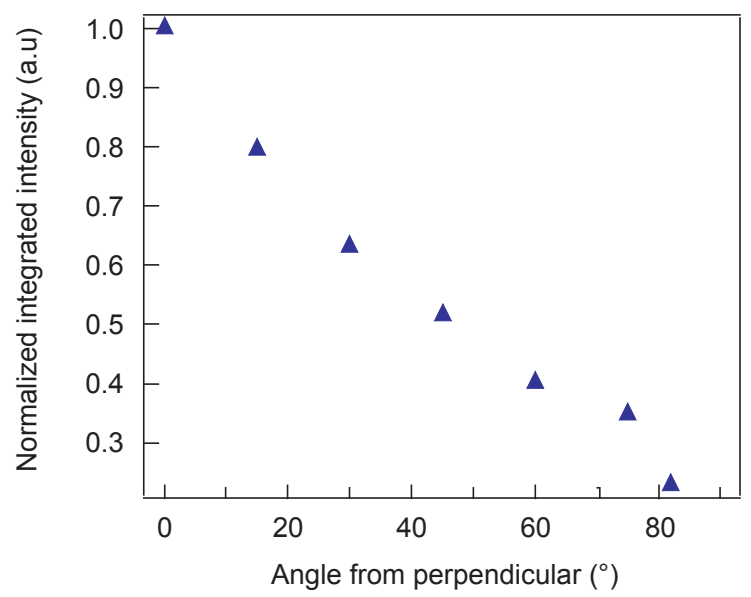

(d)

Figure 4 (a) Schematic view of waveguiding experimental setup. (b) Electroluminescence intensity captured by CCD camera at $25 \vee$ forward bias. (c) Electroluminescence spectra at various angles from vertical orientation of the nanowire array. (d) Normalized integrated intensity of EL emission as a function of angle from the perpendicular

a current injection of $200 \mathrm{~mA}$. However, our device geometry is different from these earlier experiments in that our waveguiding array is in fact part of the diode rather than an auxiliary component and therefore there is minimal loss of generated emission.

\section{Conclusions}

In conclusion, we have demonstrated a nanowirebased light emitting diode consisting of a p-type GaN thin film and an n-type $\mathrm{ZnO}$ vertical nanowire array. A low temperature solution-based growth procedure was utilized to produce a vertical and homogeneous array of epitaxially grown $\mathrm{ZnO}$ nanowires directly from the $\mathrm{p}-\mathrm{GaN}$ thin film. The resulting devices exhibited diode like current-voltage behavior and electroluminescence visible to the human eye at a forward bias of $10 \mathrm{~V}$.
EL was dominated by acceptor to band transitions in the $\mathrm{p}-\mathrm{GaN}$ thin film with contributions from band to band transitions and defect transitions in the nanowires. Finally, preliminary evidence was obtained for possible waveguided emission due to the unique nanowire array structure of the device.

\section{Acknowledgements}

This work was supported in part by the U.S. Department of Energy and DARPA-UPR. Work at the Lawrence Berkeley National Laboratory was supported by the Office of Science, Basic Energy Sciences, and Division of Materials Science of the U.S. Department of Energy. EL thanks Sandia National Laboratories for financial support through an educational fellowship.

\section{国然 Springer}




\section{References}

[1] Strite, S.; Morkoç, H. GaN, AIN, and InN: A review. J. Vac. Sci. Technol. B 1992, 10, 1237-1266.

[2] Wang, Z. L. Zinc oxide nanostructures: Growth, properties and applications. J. Phys.: Condens. Mat. 2004, 16, R829R858.

[3] Sirbuly, D. J.; Law, M.; Yan, J.; Yang, P. Semiconductor nanowires for subwavelength photonics integration. J. Phys. Chem. B 2005, 109, 15190-15213.

[4] Duan, X.; Huang, Y.; Cui, Y.; Wang, J.; Lieber, C. M. Indium phosphide nanowires as building blocks for nanoscale electronic and optoelectronic devices. Nature 2001, 409, 66-69.

[5] Khan, A.; Balakrishnan, K.; Katona, T. Ultraviolet lightemitting diodes based on group three nitrides. Nat. Photon. 2008, 2, 77-84.

[6] Pearton, S. J.; Ren, F.; Zhang, A. P.; Lee, K. P. Fabrication and performance of GaN electronic devices. Mater. Sci. Eng. $R$ 2000, 30, 55-212.

[7] Greene, L. E.; Law, M.; Goldberger, J.; Kim, F.; Johnson, J. C.; Zhang, Y.; Saykally, R. J.; Yang, P. Low-temperature nanowire arrays. Angew. Chem. Int. Ed. 2003, 42, 30313034.

[8] Ohgaki, T.; Sugimura, S.; Ryoken, H.; Ohashi, N.; Sakaguchi, I.; Sekiguchi, T.; Haneda, H. Interfacial structure of $\mathrm{GaN}$ and $\mathrm{InN}$ thin films grown on $\mathrm{ZnO}$ substrates. Key Eng. Mat. 2006, 301, 79-82.

[9] Johnson, J.; Yan, H.; Schaller, R. D.; Haber, L. H.; Saykally, R. J.; Yang, P. Single nanowire lasers. J. Phys. Chem. B 2001, 105, 11387-11390.

[10] Law, M.; Sirbuly, D. J.; Johnson, J. C.; Goldberger, J.; Saykally, R. J.; Yang, P. Nanoribbon waveguides for subwavelength photonics integration. Science 2004, 305, 1269-1272.

[11] Park, W. I.; Yi, G. C. Electroluminescence in $\mathrm{n}-\mathrm{ZnO}$ nanorod arrays vertically grown on $\mathrm{p}-\mathrm{GaN}$. Adv. Mater. 2004, 16, 87-90.

[12] Kim, D. C.; Han, W. S.; Kong, B. H.; Cho, H. K.; Hong, C. $\mathrm{H}$. Fabrication of the hybrid ZnO LED structure grown on p-type GaN by metal organic chemical vapor deposition. Physica B 2007, 401-402, 386-390.

[13] Jeong, M. C.; Oh, B. Y.; Ham, M. H.; Lee, S. W.; Myoung, H. M. ZnO-nanowire-inserted GaN/ZnO heterojunction light-emitting diodes. Small 2007, 3, 568-572.
[14] Sun, H.; Zhang, Q. F.; Wu, J. L. Electroluminescence from $\mathrm{ZnO}$ nanorods with an $\mathrm{n}-\mathrm{ZnO} / \mathrm{p}$-Si heterojunction structure. Nanotechnology 2006, 17, 2271-2274.

[15] Könenkamp, R.; Word, R. C.; Godinez, M. Ultraviolet electroluminescence from $\mathrm{ZnO} /$ polymer heterojunction light-emitting diodes. Nano Lett. 2005, 5, 2005-2008.

[16] Chang, C. Y.; Tsao, F. C.; Pan, C. J.; Chi, G. C.; Wang, H. T.; Chen, J. J.; Ren, F.; Norton, D. P.; Pearton, S. J.; Chen, K. $\mathrm{H}$.; Chen, L. C. Electroluminescence from ZnO nanowire/ polymer composite $\mathrm{p}-\mathrm{n}$ junction. Appl. Phys. Lett. 2006, 88, 173503.

[17] Nadarajah, A.; Word, R. C.; Meiss, J.; Könenkamp, R. Flexible inorganic nanowire light-emitting diode. Nano Lett. 2008, 8, 534-537.

[18] Yang, W. Q.; Huo, H. B.; Dai, L.; Ma, R. M.; Liu, S. F.; Ran, G. Z.; Shen, B.; Lin, C. L.; Qin, G. G. Electrical transport and electroluminescence properties of $\mathrm{n}-\mathrm{ZnO}$ single nanowires. Nanotechnology 2006, 17, 4868-4872.

[19] Lee, S. K.; Kim, T. H.; Lee, S. Y.; Choi, K. C.; Yang, P. High-brightness gallium nitride nanowire UV-blue light emitting diodes. Philos. Mag. 2007, 87, 2105-2115.

[20] Motayed, A.; Davydov, A. V.; He, M.; Mohammad, S. N.; Melngailis, J. $365 \mathrm{~nm}$ operation of $\mathrm{n}$-nanowire/p-gallium nitride homojunction light emitting diodes. Appl. Phys. Lett. 2007, 90, 183120

[21] Zimmler, M. A.; Bao, J.; Shalish, I.; Yi, W.; Yoon, J.; Narayanamurti, V.; Capasso, F. Electroluminescence from single nanowires by tunnel injection: An experimental study. Nanotechnology 2007, 18, 1-5.

[22] Viswanath, A. K.; Shin, E. J.; Lee, J. I.; Yu, S.; Kim, D.; Kim, B.; Choi, Y.; Hong, C. H. Magnesium acceptor levels in GaN studies by photoluminescence. J. Appl. Phys. 1998, 83, 2272-2275.

[23] Johnson, J. C.; Yan, H.; Yang, P.; Saykally, R. J. Optical cavity effects in $\mathrm{ZnO}$ nanowire lasers and waveguides. J. Phys. Chem. B 2003, 107, 8816-8828.

[24] An, S. J.; Chae, J. H.; Yi, G. C.; Park, G. H. Enhanced light output of GaN-based light-emitting diodes with ZnO nanorod arrays. Appl. Phys. Lett. 2008, 92, 121108.

[25] Chiu, C. H.; Lee, C. E.; Chao, C. L.; Cheng, B. S.; Huang, H. W.; Kuo, H. C.; Lu, T. C.; Wang, S. C.; Kuo, W. L. Hsiao, C. S.; Chen, S. Y. Enhancement of light output intensity by integrating $\mathrm{ZnO}$ nanorod arrays on GaNbased LLO vertical LEDs. Electrochem. Solid-State Lett. 2008, 11, H84-H87. 